\title{
On the seventh Lie dimension subgroups
}

\author{
By Ken-Ichi Tahara and Jian XIaO \\ (Received October 22, 1992)
}

(Revised March 30, 1993)

\section{$\S 1$. Introduction}

Let $G$ be a group with the lower central series $G=G_{1} \supset G_{2} \supset \cdots \supset G_{n} \supset$ $G_{n+1} \supset \cdots$. Let $\mathbf{Z} G$ be the group ring of $G$ over $\mathbf{Z}$, the ring of rational integers, and $\Delta(G)$ the augmentation ideal of $\mathbf{Z} G$. Define the Lie powers of $\Delta(G)$ as $\Delta^{(1)}(G)=\Delta(G)$ and, inductively, $\Delta^{(n)}(G)=\left(\Delta^{(n-1)}(G), \Delta(G)\right) \mathbf{Z} G$, where $\left(\Delta^{(n-1)}(G), \Delta(G)\right)$ is the Lie bracket defined by

$$
(\alpha, \beta)=\alpha \beta-\beta \alpha, \quad \alpha \in \Delta^{(n-1)}(G), \quad \beta \in \Delta(G) .
$$

The Lie powers $\Delta^{(n)}(G)$ of $\Delta(G)$ define the $n$-th Lie dimension subgroup $D_{(n)}(G)$ of $G$ :

$$
D_{(n)}(G)=G \cap\left(1+\Delta^{(n)}(G)\right) .
$$

Sandling [4] showed $D_{(n)}(G)=G_{n}$ for any $n$ with $1 \leqq n \leqq 6$, and Hurley and Sehgal [2] constructed a group $G$ such that $D_{(n)}(G) \neq G_{n}$ for all $n$ with $n \geqq 9$ by modifying the Gupta group [1], and hence the question remains open for $n=7$ and 8 .

In this paper we show that the exponent of $D_{(7)}(G) / G_{7}$ divides 2 for any finite group $G$.

\section{§2. Notation and preliminary results}

Let $c, d$ be natural integers. We denote by $(c, d)$ the greatest common divisor of $c$ and $d$, and put

$$
\left(\begin{array}{l}
d \\
c
\end{array}\right)=d(d-1) \cdots(d-c+1) / c(c-1) \cdots 1 .
$$

Let $A$ be an abelian group. Then $\operatorname{Sp}^{n}(A)=A^{\otimes n} / J$, where $A^{\otimes n}$ is the $n$ th tensor product of $A$, and $J$ is the subgroup of $A^{\otimes n}$ generated by all elements $x_{1} \otimes x_{2} \otimes \cdots \otimes x_{n}-x_{\sigma(1)} \otimes x_{\sigma(2)} \otimes \cdots \otimes x_{\sigma(n)}$ for $x_{1}, x_{2}, \ldots, x_{n} \in A$, and $\sigma \in S_{n}$, the symmetric group of degree $n$. Denote by $x_{1} \vee x_{2} \vee \cdots \vee x_{n}$ the class of $\operatorname{Sp}^{n}(A)$ 
to which $x_{1} \otimes x_{2} \otimes \cdots \otimes x_{n}$ belongs, and by $\stackrel{n}{\vee} x$ the class $x \vee x \vee \cdots \vee x$ ( $n$ times). In particular, put $\operatorname{Sp}^{0}(A)=\mathbf{Z}$.

Following Losey [3], we introduce a uniqueness basis for a finite group. Let $G$ be a finite group with the lower central series $G=G_{1} \supset G_{2} \supset \cdots \supset G_{c} \supset G_{c+1}=1$. The lower central series induces a new weight function $w^{*}$ on $G^{\prime}=G_{2}$ : for any element $g$ of $G^{\prime}$,

$$
w^{*}(g)= \begin{cases}k-1 & \left(g \in G_{k}-G_{k+1}\right) \\ \infty & (g=1)\end{cases}
$$

We note $w^{*}(g) \geqq 1$ for any $g \in G^{\prime}$. If $g \neq 1$ is an element of $G^{\prime}$, define $o^{*}(g)$ to be the order of the coset $\bar{g}=g G_{w^{*}(g)+2}$ in $G_{w^{*}(g)+1} / G_{w^{*}(g)+2}$. Each quotient group $G_{i} / G_{i+1}$ is a finite abelian group whose operation is denoted additively, and hence there exist elements $x_{i 1}, x_{i 2}, \ldots, x_{i \lambda(i)}$ with $\bar{x}_{i j}=x_{i j} G_{i+1}$ such that any element $\bar{g} \in G_{i} / G_{i+1}$ can be uniquely written in the form

$$
\bar{g}=u(1) \bar{x}_{i l}+u(2) \bar{x}_{i 2}+\cdots+u(\lambda(i)) \bar{x}_{i \lambda(i)},
$$

where $0 \leqq u(j)<o^{*}\left(x_{i j}\right)$ for all $j$. Moreover, we choose $x_{i j}$ so that $o^{*}\left(x_{i j}\right)$ divides $o^{*}\left(x_{i j+1}\right)$ for all $j$. Set

$$
\Phi=\left\{x_{i j} \mid i=2,3, \ldots, c ; j=1,2, \ldots, \lambda(i)\right\} .
$$

Order $\Phi$ by setting $x_{i j}<x_{k l}$ if $i<k$ or $i=k$ and $j<l$. Then every element $g \in G^{\prime}$ can be uniquely written in the form

$$
g=\prod_{i=2}^{c} x_{i 1}^{\alpha_{i 1}} x_{i 2}^{\alpha_{i 2}} \cdots x_{i \lambda(i)}^{\alpha_{i \lambda(i)}}
$$

for some integers $\alpha_{i j}$ with $0 \leqq \alpha_{i j}<o^{*}\left(x_{i j}\right), 2 \leqq i \leqq c, 1 \leqq j \leqq \lambda(i)$, where $\prod$ runs in the order of increasing $i$ from left to right. Put $m=\sum_{i=2}^{c} \lambda(i)$, and consider an $m$-sequence $\alpha=\left(\alpha_{i j}\right)=\left(\alpha_{21}, \ldots, \alpha_{2 \lambda(2)}, \alpha_{31}, \ldots, \alpha_{3 \lambda(3)}, \ldots, \alpha_{c 1}, \ldots, \alpha_{c \lambda(c)}\right)$ for non-negative integers $\alpha_{i j}$. The set of all $m$-sequences is naturally well ordered, that is, we define $\alpha=\left(\alpha_{i j}\right)<\beta=\left(\beta_{i j}\right)$ if there exist $k$ and $l$ with $2 \leqq k \leqq c$ and $1 \leqq j \leqq \lambda(k)$ such that $\alpha_{i j}=\beta_{i j}(2 \leqq i \leqq k-1,1 \leqq j \leqq \lambda(i)), \alpha_{k j}=\beta_{k j}$ $(1 \leqq j \leqq l-1)$, and $\alpha_{k l}<\beta_{k l}$. An $m$-sequence $\alpha=\left(\alpha_{i j}\right)$ is basic if $0 \leqq \alpha_{i j}<o^{*}\left(x_{i j}\right)$ for all $i$ and $j$. It follows from the uniqueness of the expression (\#) that there is a bijection between the elements of $G^{\prime}$ and the basic $m$-sequences. The weight $W^{*}$ of an $m$-sequence $\alpha=\left(\alpha_{i j}\right)$ is defined to be

$$
W^{*}(\alpha)=\sum_{\substack{2 \leqq i \leqq c \\ 1 \leqq j \leqq \lambda(i)}} w^{*}\left(x_{i j}\right) \alpha_{i j}=\sum_{2 \leqq i \leqq c}(i-1)\left(\sum_{\substack{1 \leqq j \leqq \lambda(i) \\ 2}} \alpha_{i j}\right) .
$$


Given an $m$-sequence $\alpha=\left(\alpha_{i j}\right)$, we define the proper product $P(\alpha) \in \mathbf{Z} G$ by

$$
P(\alpha)=\prod_{i=2}^{c}\left(x_{i 1}-1\right)^{\alpha_{i 1}}\left(x_{i 2}-1\right)^{\alpha_{i 2}} \cdots\left(x_{i \lambda(i)}-1\right)^{\alpha_{i \lambda(i)}}
$$

where $\prod$ runs in the order of increasing $i$ from left to right. If $\alpha$ is a basic $m$ sequence, then $P(\alpha)$ is called a basic product. We introduce notation for cutting an element $g$ of $G^{\prime}$ modulo the $i$-th subgroup $G_{i}$. Let $P_{i}(g)$ be the product of weight $i-1$ in the expression (\#):

$$
P_{i}(g)=x_{i 1}^{\alpha_{i 1}} x_{i 2}^{\alpha_{i 2}} \cdots x_{i \lambda(i)}^{\alpha_{i \lambda(i)}},
$$

and $\bar{P}_{i}(g)$ the coset of $P_{i}(g)$ modulo $G_{i+1}$ :

$$
\bar{P}_{i}(g)=\sum_{j=1}^{\lambda(i)} \alpha_{i j} \bar{x}_{i j} \in G_{i} / G_{i+1} .
$$

On the other hand, we put

$$
W_{(m)}=W_{(m)}(G)=\sum \bigotimes_{i=1}^{m} \operatorname{Sp}^{a_{i}}\left(G_{i} / G_{i+1}\right),
$$

where $\sum$ runs over all non-negative integers $a_{1}, a_{2}, \ldots, a_{m}$ such that $\sum_{i=2}^{m}(i-1) a_{i}=$ $m-1$. In particular,

$$
\begin{aligned}
W_{(4)}= & \operatorname{Sp}^{3}\left(G_{2} / G_{3}\right) \oplus\left(G_{2} / G_{3} \otimes G_{3} / G_{4}\right) \oplus G_{4} / G_{5} \\
W_{(5)}= & \operatorname{Sp}^{4}\left(G_{2} / G_{3}\right) \oplus\left(\operatorname{Sp}^{2}\left(G_{2} / G_{3}\right) \otimes G_{3} / G_{4}\right) \oplus\left(G_{2} / G_{3} \otimes G_{4} / G_{5}\right) \\
& \oplus \operatorname{Sp}^{2}\left(G_{3} / G_{4}\right) \oplus G_{5} / G_{6} \\
W_{(6)}= & \operatorname{Sp}^{5}\left(G_{2} / G_{3}\right) \oplus\left(\operatorname{Sp}^{3}\left(G_{2} / G_{3}\right) \otimes G_{3} / G_{4}\right) \oplus\left(\mathrm{Sp}^{2}\left(G_{2} / G_{3}\right) \otimes G_{4} / G_{5}\right) \\
& \oplus\left(G_{2} / G_{3} \otimes \operatorname{Sp}^{2}\left(G_{3} / G_{4}\right)\right) \oplus\left(G_{2} / G_{3} \otimes G_{5} / G_{6}\right) \\
& \oplus\left(G_{3} / G_{4} \otimes G_{4} / G_{5}\right) \oplus G_{6} / G_{7} .
\end{aligned}
$$

We fix the set of representatives $t$ of $G$ with respect to $G^{\prime}=G_{2}$, namely

$$
G=\bigcup_{t \in T} t G_{2}
$$

Denote $a(i j)=o^{*}\left(x_{i j}\right)(2 \leqq i \leqq 6,1 \leqq j \leqq \lambda(i))$. Moreover put

$$
x_{i j}^{a(i j)} \equiv \prod_{k=1}^{\lambda(i+1)} x_{i+1 k}^{a(i j k)} \cdot \prod_{l=1}^{\lambda(i+2)} x_{i+2 l}^{a^{\prime}(i j l)} \bmod G_{i+3}, \quad 2 \leqq i \leqq 5, \quad 1 \leqq j \leqq \lambda(i)
$$




$$
\begin{aligned}
& {\left[x_{2 i}^{a(2 i)}, x_{2 j}\right] \equiv \prod_{k=1}^{\lambda(5)} x_{5 k}^{\alpha_{k}(i j)} \cdot \prod_{l=1}^{\lambda(6)} x_{6 l}^{\alpha_{l}(i j)^{\prime}} \bmod G_{7}, \quad 1 \leqq i<j \leqq \lambda(2)} \\
& {\left[x_{3 i}, x_{3 j}\right] \equiv \prod_{k=1}^{\lambda(6)} x_{6 k}^{\delta_{k}^{(i j)}} \bmod G_{7}, \quad 1 \leqq i<j \leqq \lambda(3) .}
\end{aligned}
$$

\section{§3. Structure of $\Delta^{(n)}(G)$ for $n=4,5,6$ and 7}

Sandling [4] showed that, for $n \geqq 2$,

$$
\Delta^{(n)}(G)=\sum \prod_{j} \Delta\left(G_{n_{j}}\right) \mathbf{Z} G
$$

where $\sum$ runs over all $n_{j}, n \geqq n_{j} \geqq 2$ with $\sum_{j}\left(n_{j}-1\right)=n-1$.

On the other hand, we can show the following:

Lemma 3.1. Let $G$ be a group with $G_{2} / G_{3}$ having finite exponent. Then we have for any $n \geqq 2$,

$$
\operatorname{rank}_{\mathbf{Z}} \Delta^{(n)}(G)=\operatorname{rank}_{\mathbf{Z}} \Delta^{(2)}(G)
$$

In particuler, if $G$ is a finite group, then for any $n \geqq 2$,

$$
\operatorname{rank}_{\mathbf{Z}} \Delta^{(n)}(G)=|G|-\left|G / G_{2}\right|=\left|G / G_{2}\right|\left(\left|G_{2}\right|-1\right) .
$$

Proof. We can easily prove the lemma in three steps by a method similar to that in Losey [3]. The first step is to show that $e \Delta^{(n)}(G)$ is contained in $\Delta^{(n+1)}(G)$ for any $n \geqq 2$ if the exponent $e$ of $G_{2} / G_{3}$ is finite, and the second one is to show that $e \Delta^{(m)}(G)$ is contained in $\Delta^{(m+2)}(G)$ for any $m \geqq 0$. The final step is to show $\operatorname{rank}_{\mathbf{Z}} \Delta^{(n+2)}(G)=\operatorname{rank}_{\mathbf{Z}} \Delta^{(2)}(G)$ by using the fact that $\Delta^{(2)}(G)$ is isomorphic to $e \Delta^{(2)}(G)$.

Q.E.D.

Using the above lemma, we have the following Lemmas $3.2,3.3$ by the same methods as in the proofs of [3, Lemma 7], [5, Lemma 4] and [6, Lemma 3.5].

Lemma 3.2. $\Delta^{(4)}(G)$ has a $\mathbf{Z}$-free basis consisting of

$$
\begin{aligned}
& t\left(x_{i j}-1\right)^{a(i j)}, \quad 2 \leqq i \leqq 3, \quad(i-1) a(i j) \geqq 3, \quad 1 \leqq j \leqq \lambda(i) \\
& t a(2 i)\left(x_{2 i}-1\right)\left(x_{2 j}-1\right), \quad 1 \leqq i \leqq j \leqq \lambda(2) \\
& t P(\alpha), \quad \alpha: \text { basic }, \quad W^{*}(\alpha) \geqq 3,
\end{aligned}
$$

for all $t \in T$. 
Lemma 3.3. For $u=5,6$ and $7, \Delta^{(u)}(G)$ has a system of $\mathbf{Z}$-generators consisting of

$$
\begin{aligned}
& \text { (1) } \quad t\left(x_{i j}-1\right)^{a(i j)}, \quad 2 \leqq i \leqq u-1, \quad(i-1) a(i j) \geqq u-1, \quad 1 \leqq j \leqq \lambda(i) \\
&(2) \quad t\left(x_{i j}-1\right)^{a(i j)}\left(x_{k l}-1\right), \quad 2 \leqq i \leqq k, \quad i+k<u, \quad(i-1) a(i j)+k \geqq u, \\
& 1 \leqq j \leqq \lambda(i), \quad 1 \leqq l \leqq \lambda(k) \\
&(3) \quad t\left(x_{i j}-1\right)\left(x_{k l}-1\right)^{a(k l)}, \quad 2 \leqq i \leqq k, \quad i+k<u, \quad(k-1) a(k l)+i \geqq u \\
& 1 \leqq j \leqq \lambda(i), \quad 1 \leqq l \leqq \lambda(k)
\end{aligned}
$$

(4) $\quad t\left(x_{i j}-1\right)^{a(i j)}\left(x_{k l}-1\right)\left(x_{m n}-1\right), \quad 2 \leqq i \leqq k \leqq m, \quad i+k+m-1<u$,

$$
\begin{aligned}
& (i-1) a(i j)+k+m-1 \geqq u, \quad 1 \leqq j \leqq \lambda(i), \\
& 1 \leqq l \leqq \lambda(k), \quad 1 \leqq n \leqq \lambda(m)
\end{aligned}
$$

$$
\begin{gathered}
t\left(x_{i j}-1\right)\left(x_{k l}-1\right)^{a(k l)}\left(x_{m n}-1\right), \quad 2 \leqq i \leqq k \leqq m, \quad i+k+m-1<u \\
(k-1) a(k l)+i+m-1 \geqq u, \quad 1 \leqq j \leqq \lambda(i), \\
1 \leqq l \leqq \lambda(k), \quad 1 \leqq n \leqq \lambda(m)
\end{gathered}
$$

(6) $\quad t\left(x_{i j}-1\right)\left(x_{k l}-1\right)\left(x_{m n}-1\right)^{a(m n)}, \quad 2 \leqq i \leqq k \leqq m, \quad i+k+m-1<u$,

$$
\begin{aligned}
& (m-1) a(m n)+i+k-1 \geqq u, \quad 1 \leqq j \leqq \lambda(i), \\
& 1 \leqq l \leqq \lambda(k), \quad 1 \leqq n \leqq \lambda(m)
\end{aligned}
$$

(7) $\quad t\left(x_{i j}-1\right)^{a(i j)}\left(x_{k l}-1\right)\left(x_{m n}-1\right)\left(x_{p q}-1\right), \quad 2 \leqq i \leqq k \leqq m \leqq p$,

$$
i+k+m+p-2<u, \quad(i-1) a(i j)+k+m+p-2 \geqq u,
$$$$
1 \leqq j \leqq \lambda(i), \quad 1 \leqq l \leqq \lambda(k), \quad 1 \leqq n \leqq \lambda(m), \quad 1 \leqq q \leqq \lambda(p)
$$

$$
\begin{aligned}
& t\left(x_{i j}-1\right)\left(x_{k l}-1\right)^{a(k l)}\left(x_{m n}-1\right)\left(x_{p q}-1\right), \quad 2 \leqq i \leqq k \leqq m \leqq p \\
& i+k+m+p-2<u, \quad(k-1) a(k l)+i+m+p-2 \geqq u, \\
& 1 \leqq j \leqq \lambda(i), \quad 1 \leqq l \leqq \lambda(k), \quad 1 \leqq n \leqq \lambda(m), \quad 1 \leqq q \leqq \lambda(p)
\end{aligned}
$$

$$
\begin{aligned}
& t\left(x_{i j}-1\right)\left(x_{k l}-1\right)\left(x_{m n}-1\right)^{a(m n)}\left(x_{p q}-1\right), \quad 2 \leqq i \leqq k \leqq m \leqq p, \\
& i+k+m+p-2<u, \quad(m-1) a(m n)+i+k+p-2 \geqq u, \\
& 1 \leqq j \leqq \lambda(i), \quad 1 \leqq l \leqq \lambda(k), \quad 1 \leqq n \leqq \lambda(m), \quad 1 \leqq q \leqq \lambda(p)
\end{aligned}
$$

(10) $\quad t\left(x_{i j}-1\right)\left(x_{k l}-1\right)\left(x_{m n}-1\right)\left(x_{p q}-1\right)^{a(p q)}, \quad 2 \leqq i \leqq k \leqq m \leqq p$,

$$
\begin{aligned}
& i+k+m+p-2<u, \quad(p-1) a(p q)+i+k+m-2 \geqq u \\
& 1 \leqq j \leqq \lambda(i), \quad 1 \leqq l \leqq \lambda(k), \quad 1 \leqq n \leqq \lambda(m), \quad 1 \leqq q \leqq \lambda(p)
\end{aligned}
$$




$$
\begin{gathered}
\text { (11) } \quad t(a(i j), a(k l))\left(x_{i j}-1\right)\left(x_{k l}-1\right), \quad 2 \leqq i \leqq k, \quad i+k=u, \\
\quad 1 \leqq j \leqq \lambda(i), \quad 1 \leqq l \leqq \lambda(k) \\
(12) \quad t(a(i j), a(k l), a(m n))\left(x_{i j}-1\right)\left(x_{k l}-1\right)\left(x_{m n}-1\right), \quad 2 \leqq i \leqq k \leqq m, \\
i+k+m-1=u, \quad 1 \leqq j \leqq \lambda(i), \quad 1 \leqq l \leqq \lambda(k), \quad 1 \leqq n \leqq \lambda(m) \\
(13) \quad t(a(i j), a(k l), a(m n), a(p q))\left(x_{i j}-1\right)\left(x_{k l}-1\right)\left(x_{m n}-1\right)\left(x_{p q}-1\right), \\
2 \leqq i \leqq k \leqq m \leqq p, \quad i+k+m+p-2=u, \\
1 \leqq j \leqq \lambda(i), \quad 1 \leqq l \leqq \lambda(k), \quad 1 \leqq n \leqq \lambda(m), \quad 1 \leqq q \leqq \lambda(p) \\
\quad t(a(i j), a(k l), a(m n), a(p q), a(r s))\left(x_{i j}-1\right)\left(x_{k l}-1\right)\left(x_{m n}-1\right)\left(x_{p q}-1\right) \\
\times\left(x_{r s}-1\right), \\
2 \leqq i \leqq k \leqq m \leqq p \leqq r, \quad i+k+m+p+r-3=u, \\
1 \leqq j \leqq \lambda(i), \quad 1 \leqq l \leqq \lambda(k), \quad 1 \leqq n \leqq \lambda(m), \\
1 \leqq q \leqq \lambda(p), \quad 1 \leqq s \leqq \lambda(r) \\
\quad t P(\alpha), \quad \alpha: b a s i c, \quad W^{*}(\alpha) \geqq u-1,
\end{gathered}
$$

for all $t \in T$.

\section{§4. Exponent of $D_{(7)}(G) / G_{7}$}

We denote by $W_{(m)}^{*}=W_{(m)}^{*}(G)$ the free group generated by a basis of $W_{(m)}=$ $W_{(m)}(G)$. Note that an element in such a basis will have finite order in $W_{(m)}$, but infinite order in $W_{(m)}^{*}$.

For simplicity in this section, put $a(i)=a(2 i)(1 \leqq i \leqq \lambda(2)), b(i)=a(3 i)$ $(1 \leqq i \leqq \lambda(3)), c(i)=a(4 i)(1 \leqq i \leqq \lambda(4)), d(i)=a(5 i)(1 \leqq i \leqq \lambda(5)), a_{i k}=a(2 i k)$ $(1 \leqq i \leqq \lambda(2), 1 \leqq k \leqq \lambda(3)), b_{i k}=a(3 i k)(1 \leqq i \leqq \lambda(3), 1 \leqq k \leqq \lambda(4)), c_{i k}=$ $a(4 i k)(1 \leqq i \leqq \lambda(4), 1 \leqq k \leqq \lambda(5)), d_{i k}=a(5 i k)(1 \leqq i \leqq \lambda(5), 1 \leqq k \leqq \lambda(6))$.

Now, we define a homomorphism $\psi: \Delta^{(4)}(G) \longrightarrow W_{(4)}^{*} \oplus W_{(5)}^{*} \oplus W_{(6)}$ by

1) $\quad \psi\left(t\left(x_{2 i}-1\right)^{a(i)}\right)=\left\{\begin{array}{ll}\bigvee^{(i)} \bar{x}_{2 i}, & 3 \leqq a(i) \leqq 5 \\ 0, & a(i) \geqq 6\end{array}, \quad 1 \leqq i \leqq \lambda(2)\right.$

2) $\quad \psi\left(t\left(x_{3 i}-1\right)^{b(i)}\right)=\left\{\begin{array}{ll}\bigvee^{b(i)} x_{3 i}, & b(i)=2 \\ 0, & b(i) \geqq 3\end{array}, \quad 1 \leqq i \leqq \lambda(3)\right.$

3) $\quad \psi\left(\operatorname{ta}(i)\left(x_{2 i}-1\right)\left(x_{2 j}-1\right)\right)$ 


$$
\begin{aligned}
& =-\sum_{h=2}^{4}\left(\begin{array}{c}
a(i) \\
h
\end{array}\right)\left(\vee^{h} \bar{x}_{2 i} \vee \bar{x}_{2 j}\right)+\left(\bar{x}_{2 j} \otimes \overline{x_{2 i}^{a(i)}}\right)+\left(\bar{x}_{2 j} \otimes \sum_{h=4}^{5} \bar{P}_{h}\left(x_{2 i}^{a(i)}\right)\right) \\
& +\left(\bar{x}_{2 j} \otimes \sum_{k=1}^{\lambda(3)}\left(\begin{array}{c}
a_{i k} \\
2
\end{array}\right) \stackrel{2}{\vee} \bar{x}_{3 k}\right)+\left(\bar{x}_{2 j} \otimes \sum_{1 \leqq k<l \leqq \lambda(3)} a_{i k} a_{i l}\left(\bar{x}_{3 k} \vee \bar{x}_{3 l}\right)\right) \\
& \left.+\left[\overline{x_{2 i}^{a(i)}, x_{2 j}}\right]+\bar{P}_{6}\left(\left[x_{2 i}^{a(i)}, x_{2 j}\right]\right)+\left(\bar{x}_{2 j} \otimes \overline{\left[x_{2 i}^{a(i)}, x_{2 j}\right.}\right]\right), \quad 1 \leqq i \leqq j \leqq \lambda(2)
\end{aligned}
$$

4) $\quad \psi\left(t\left(x_{2 i}-1\right)\left(x_{2 j}-1\right)\left(x_{2 k}-1\right)\right)=\bar{x}_{2 i} \vee \bar{x}_{2 j} \vee \bar{x}_{2 k}$,

$$
\left(x_{2 i}-1\right)\left(x_{2 j}-1\right)\left(x_{2 k}-1\right): \text { basic }, \quad 1 \leqq i \leqq j \leqq k \leqq \lambda(2)
$$

5) $\quad \psi\left(t\left(x_{2 i}-1\right)\left(x_{3 j}-1\right)\right)=\bar{x}_{2 i} \otimes \bar{x}_{3 j}, \quad 1 \leqq i \leqq \lambda(2), \quad 1 \leqq j \leqq \lambda(3)$

6) $\quad \psi\left(t\left(x_{4 i}-1\right)\right)=\bar{x}_{4 i}, \quad 1 \leqq i \leqq \lambda(4)$

7) $\quad \psi\left(t\left(x_{2 i}-1\right)\left(x_{2 j}-1\right)\left(x_{2 k}-1\right)\left(x_{2 l}-1\right)\right)=\bar{x}_{2 i} \vee \bar{x}_{2 j} \vee \bar{x}_{2 k} \vee \bar{x}_{2 l}$,

$$
\left(x_{2 i}-1\right)\left(x_{2 j}-1\right)\left(x_{2 k}-1\right)\left(x_{2 l}-1\right): \text { basic, } \quad 1 \leqq i \leqq j \leqq k \leqq l \leqq \lambda(2)
$$

8) $\quad \psi\left(t\left(x_{2 i}-1\right)\left(x_{2 j}-1\right)\left(x_{3 k}-1\right)\right)=\left(\bar{x}_{2 i} \vee \bar{x}_{2 j}\right) \otimes \bar{x}_{3 k}$,

$$
\left(x_{2 i}-1\right)\left(x_{2 j}-1\right): \text { basic }, \quad 1 \leqq i \leqq j \leqq \lambda(2), \quad 1 \leqq k \leqq \lambda(3)
$$

9) $\quad \psi\left(t\left(x_{2 i}-1\right)\left(x_{4 j}-1\right)\right)=\bar{x}_{2 i} \otimes \bar{x}_{4 j}, \quad 1 \leqq i \leqq \lambda(2), \quad 1 \leqq j \leqq \lambda(4)$

10) $\psi\left(t\left(x_{3 i}-1\right)\left(x_{3 j}-1\right)\right)=\bar{x}_{3 i} \vee \bar{x}_{3 j}, \quad\left(x_{3 i}-1\right)\left(x_{3 j}-1\right):$ basic,

11) $\psi\left(t\left(x_{5 i}-1\right)\right)=\bar{x}_{5 i}, \quad 1 \leqq i \leqq \lambda(5)$

12) $\psi\left(t\left(x_{2 i}-1\right)\left(x_{2 j}-1\right)\left(x_{2 k}-1\right)\left(x_{2 l}-1\right)\left(x_{2 m}-1\right)\right)$

$$
=\bar{x}_{2 i} \vee \bar{x}_{2 j} \vee \bar{x}_{2 k} \vee \bar{x}_{2 l} \vee \bar{x}_{2 m} \text {, }
$$

$$
\begin{aligned}
& \left(x_{2 i}-1\right)\left(x_{2 j}-1\right)\left(x_{2 k}-1\right)\left(x_{2 l}-1\right)\left(x_{2 m}-1\right): \text { basic, } \\
& 1 \leqq i \leqq j \leqq k \leqq l \leqq m \leqq \lambda(2)
\end{aligned}
$$

13) $\psi\left(t\left(x_{2 i}-1\right)\left(x_{2 j}-1\right)\left(x_{2 k}-1\right)\left(x_{3 l}-1\right)\right)=\left(\bar{x}_{2 i} \vee \bar{x}_{2 j} \vee \bar{x}_{2 k}\right) \otimes \bar{x}_{3 l}$,

$$
\left(x_{2 i}-1\right)\left(x_{2 j}-1\right)\left(x_{2 k}-1\right): \text { basic }, \quad 1 \leqq i \leqq j \leqq k \leqq \lambda(2), \quad 1 \leqq l \leqq \lambda(3)
$$

14) $\psi\left(t\left(x_{2 i}-1\right)\left(x_{2 j}-1\right)\left(x_{4 k}-1\right)\right)=\left(\bar{x}_{2 i} \vee \bar{x}_{2 j}\right) \otimes \bar{x}_{4 k}$,

$$
\left(x_{2 i}-1\right)\left(x_{2 j}-1\right): \text { basic }, \quad 1 \leqq i \leqq j \leqq \lambda(2), \quad 1 \leqq k \leqq \lambda(4)
$$

15) $\psi\left(t\left(x_{2 i}-1\right)\left(x_{3 j}-1\right)\left(x_{3 k}-1\right)\right)=\bar{x}_{2 i} \otimes\left(\bar{x}_{3 j} \vee \bar{x}_{3 k}\right)$,

$$
\left(x_{3 j}-1\right)\left(x_{3 k}-1\right): \text { basic }, \quad 1 \leqq i \leqq \lambda(2), \quad 1 \leqq j \leqq k \leqq \lambda(3)
$$


16) $\quad \psi\left(t\left(x_{2 i}-1\right)\left(x_{5 j}-1\right)\right)=\bar{x}_{2 i} \otimes \bar{x}_{5 j}, \quad 1 \leqq i \leqq \lambda(2), \quad 1 \leqq j \leqq \lambda(5)$

17) $\psi\left(t\left(x_{3 i}-1\right)\left(x_{4 j}-1\right)\right)=\bar{x}_{3 i} \otimes \bar{x}_{4 j}, \quad 1 \leqq i \leqq \lambda(3), \quad 1 \leqq j \leqq \lambda(4)$

18) $\quad \psi\left(t\left(x_{6 i}-1\right)\right)=\bar{x}_{6 i}, \quad 1 \leqq i \leqq \lambda(6)$

19) $\quad \psi(t P(\alpha))=0, \quad \alpha$ : basic,$\quad W^{*}(\alpha) \geqq 6$.

We denote by $R$ the subgroup of $W_{(4)}^{*} \oplus W_{(5)}^{*} \oplus W_{(6)}^{*}$ generated by the elements of the following types (I) (IX):

$$
\begin{aligned}
& \gamma_{i}=c(i) \bar{x}_{4 i}-\overline{x_{4 i}^{c(i)}}-\bar{P}_{6}\left(x_{4 i}^{c(i)}\right), \quad 1 \leqq i \leqq \lambda(4) \\
& \gamma_{i}^{\prime}=d(i) \bar{x}_{5 i}-\overline{x_{5 i}^{d(i)}}, \quad 1 \leqq i \leqq \lambda(5) \\
& \gamma_{i j}=\sum_{h=2}^{4}\left\{\frac{a(j)}{a(i)}\left(\begin{array}{c}
a(i) \\
h
\end{array}\right)\left(\stackrel{h}{\vee} \bar{x}_{2 i} \vee \bar{x}_{2 j}\right)-\left(\begin{array}{c}
a(j) \\
h
\end{array}\right)\left(\bar{x}_{2 i} \stackrel{h}{\vee} \bar{x}_{2 j}\right)\right\} \\
& -\frac{a(j)}{a(i)}\left(\bar{x}_{2 j} \otimes \overline{x_{2 i}^{a(i)}}\right)-\frac{a(j)}{a(i)} \sum_{h=4}^{5}\left(\bar{x}_{2 j} \otimes \bar{P}_{h}\left(x_{2 i}^{a(i)}\right)\right) \\
& -\frac{a(j)}{a(i)}\left(\bar{x}_{2 j} \otimes \sum_{k=1}^{\lambda(2)}\left(\begin{array}{c}
a_{i k} \\
2
\end{array}\right) \stackrel{2}{\vee} \bar{x}_{3 k}\right) \\
& -\frac{a(j)}{a(i)}\left(\bar{x}_{2 j} \otimes \sum_{1 \leqq k<l \leqq \lambda(3)} a_{i k} a_{i l}\left(\bar{x}_{3 k} \vee \bar{x}_{3 l}\right)\right)+\left(\bar{x}_{2 i} \otimes \overline{x_{2 j}^{a(j)}}\right) \\
& +\sum_{h=4}^{5}\left(\bar{x}_{2 i} \otimes \bar{P}_{h}\left(x_{2 j}^{a(j)}\right)\right)+\left(\bar{x}_{2 i} \otimes \sum_{1 \leqq k<l \leqq \lambda(3)} a_{j k} a_{j l}\left(\bar{x}_{3 k} \vee \bar{x}_{3 l}\right)\right) \\
& +\left(\bar{x}_{2 i} \otimes \sum_{k=1}^{\lambda(3)}\left(\begin{array}{c}
a_{j k} \\
2
\end{array}\right) \stackrel{2}{\vee}^{2} \bar{x}_{3 k}\right)-\frac{a(j)}{a(i)}\left\{\overline{\left[x_{2 i}^{a(i)}, x_{2 j}\right.}\right] \\
& \left.+\bar{P}_{6}\left(\left[x_{2 i}^{a(i)}, x_{2 j}\right]\right)+\left(\bar{x}_{2 j} \otimes\left[\overline{x_{2 i}^{a(i)}, x_{2 j}}\right]\right)\right\}, \quad 1 \leqq i \leqq j \leqq \lambda(2)
\end{aligned}
$$

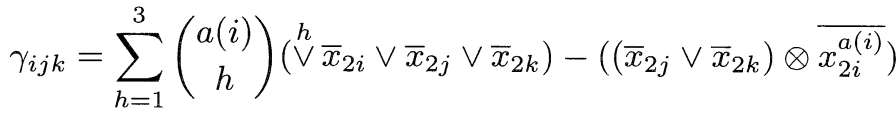

$$
\begin{aligned}
& -\left(\left(\bar{x}_{2 j} \vee \bar{x}_{2 k}\right) \otimes \bar{P}_{4}\left(x_{2 i}^{a(i)}\right)\right)-\left(\bar{x}_{2 j} \otimes\left[\overline{x_{2 i}^{a(i)}, x_{2 k}}\right]\right) \\
& -\left(\bar{x}_{2 k} \otimes\left[\overline{x_{2 i}^{a(i)}, x_{2 j}}\right]\right), \quad 1 \leqq i \leqq j \leqq k \leqq \lambda(2)
\end{aligned}
$$




$$
\begin{aligned}
\widetilde{\gamma}_{i j k}= & \sum_{h=1}^{3}\left(\begin{array}{c}
a(j) \\
h
\end{array}\right)\left(\bar{x}_{2 i} \stackrel{h}{ }^{\left.x_{2 j} \vee \bar{x}_{2 k}\right)-\left(\left(\bar{x}_{2 i} \vee \bar{x}_{2 k}\right) \otimes \overline{x_{2 j}^{a(j)}}\right.}\right) \\
& \left.-\left(\left(\bar{x}_{2 i} \vee \bar{x}_{2 k}\right) \otimes \bar{P}_{4}\left(x_{2 j}^{a(j)}\right)\right)-\left(\bar{x}_{2 i} \otimes \overline{\left[x_{2 j}^{a(j)}, x_{2 k}\right.}\right]\right)
\end{aligned}
$$

$$
1 \leqq i<j \leqq k \leqq \lambda(2)
$$

$$
\begin{aligned}
\widetilde{\widetilde{\gamma}}_{i j k}= & \sum_{h=1}^{3}\left(\begin{array}{c}
a(k) \\
h
\end{array}\right)\left(\bar{x}_{2 i} \vee \bar{x}_{2 j} \vee{ }^{h} \bar{x}_{2 k}\right)-\left(\left(\bar{x}_{2 i} \vee \bar{x}_{2 j}\right) \otimes \overline{x_{2 k}^{a(k)}}\right) \\
& -\left(\left(\bar{x}_{2 i} \vee \bar{x}_{2 j}\right) \otimes \bar{P}_{4}\left(x_{2 k}^{a(k)}\right)\right), \quad 1 \leqq i \leqq j<k \leqq \lambda(2)
\end{aligned}
$$

$$
\varepsilon_{i j k l}=\sum_{h=1}^{2}\left(\begin{array}{c}
a(i) \\
h
\end{array}\right)\left(\stackrel{h}{ }^{\prime} \bar{x}_{2 i} \vee \bar{x}_{2 j} \vee \bar{x}_{2 k} \vee \bar{x}_{2 l}\right)-\left(\left(\bar{x}_{2 j} \vee \bar{x}_{2 k} \vee \bar{x}_{2 l}\right) \otimes \overline{x_{2 i}^{a(i)}}\right) \text {, }
$$

$$
1 \leqq i \leqq j \leqq k \leqq l \leqq \lambda(2)
$$

$$
\widetilde{\varepsilon}_{i j k l}=\sum_{h=1}^{2}\left(\begin{array}{c}
a(j) \\
h
\end{array}\right)\left(\bar{x}_{2 i} \vee \bar{x}_{2 j} \vee \bar{x}_{2 k} \vee \bar{x}_{2 l}\right)-\left(\left(\bar{x}_{2 i} \vee \bar{x}_{2 k} \vee \bar{x}_{2 l}\right) \otimes \overline{x_{2 j}^{a(j)}}\right),
$$$$
1 \leqq i<j \leqq k \leqq l \leqq \lambda(2)
$$

$$
\widetilde{\widetilde{\varepsilon}}_{i j k l}=\sum_{h=1}^{2}\left(\begin{array}{c}
a(k) \\
h
\end{array}\right)\left(\bar{x}_{2 i} \vee \bar{x}_{2 j} \vee \bar{x}_{2 k} \vee \bar{x}_{2 l}\right)-\left(\left(\bar{x}_{2 i} \vee \bar{x}_{2 j} \vee \bar{x}_{2 l}\right) \otimes \overline{x_{2 k}^{a(k)}}\right),
$$

$$
1 \leqq i \leqq j<k \leqq l \leqq \lambda(2)
$$

$$
\begin{array}{r}
\widetilde{\widetilde{\varepsilon}}_{i j k l}=\sum_{h=1}^{2}\left(\begin{array}{c}
a(l) \\
h
\end{array}\right)\left(\bar{x}_{2 i} \vee \bar{x}_{2 j} \vee \bar{x}_{2 k} \vee \bar{x}_{2 l}\right)-\left(\left(\bar{x}_{2 i} \vee \bar{x}_{2 j} \vee \bar{x}_{2 k}\right) \otimes \overline{x_{2 l}^{a(l)}}\right), \\
1 \leqq i \leqq j \leqq k<l \leqq \lambda(2)
\end{array}
$$

(VI)

$$
\begin{aligned}
& \delta_{i j}= \sum_{h=1}^{3}\left(\begin{array}{c}
a(i) \\
h
\end{array}\right)\left(\stackrel{h}{\vee} \bar{x}_{2 i} \otimes \bar{x}_{3 j}\right)-\left(\overline{x_{2 i}^{a(i)}} \vee \bar{x}_{3 j}\right)-\left(\bar{x}_{3 j} \otimes \bar{P}_{4}\left(x_{2 i}^{a(i)}\right)\right) \\
&-\sum_{1 \leqq j<h \leqq \lambda(3)} a_{i h}\left[\overline{x_{3 h}, x_{3 j}}\right], \quad 1 \leqq i \leqq \lambda(2), \quad 1 \leqq j \leqq \lambda(3) \\
& \widetilde{\delta}_{i j}= \sum_{h=1}^{2}\left(\begin{array}{c}
b(j) \\
h
\end{array}\right)\left(\bar{x}_{2 i} \otimes \vee \bar{x}_{3 j}\right)-\left(\bar{x}_{2 i} \otimes \overline{x_{3 j}^{b(j)}}\right)-\left(\bar{x}_{2 i} \otimes \bar{P}_{5}\left(x_{3 j}^{b(j)}\right)\right), \\
& 1 \leqq i \leqq \lambda(2), \quad 1 \leqq j \leqq \lambda(3)
\end{aligned}
$$


(VII)

$$
\begin{aligned}
& \theta_{i j k}=\sum_{h=1}^{2}\left(\begin{array}{c}
a(i) \\
h
\end{array}\right)\left(\left(\vee^{h} \bar{x}_{2 i} \vee \bar{x}_{2 j}\right) \otimes \bar{x}_{3 k}\right)-\left(\bar{x}_{2 j} \otimes\left(\bar{x}_{3 k} \vee \overline{x_{2 i}^{a(i)}}\right)\right) \\
& 1 \leqq i \leqq j \leqq \lambda(2), \quad 1 \leqq k \leqq \lambda(3) \\
& \tilde{\theta}_{i j k}=\sum_{h=1}^{2}\left(\begin{array}{c}
a(j) \\
h
\end{array}\right)\left(\left(\bar{x}_{2 i} \stackrel{h}{\vee} \bar{x}_{2 j}\right) \otimes \bar{x}_{3 k}\right)-\left(\bar{x}_{2 i} \otimes\left(\bar{x}_{3 k} \vee \overline{x_{2 j}^{a(j)}}\right)\right), \\
& 1 \leqq i<j \leqq \lambda(2), \quad 1 \leqq k \leqq \lambda(3) \\
& \widetilde{\widetilde{\theta}}_{i j k}=b(k)\left(\left(\bar{x}_{2 i} \vee \bar{x}_{2 j}\right) \otimes \bar{x}_{3 k}\right)-\left(\left(\bar{x}_{2 i} \vee \bar{x}_{2 j}\right) \otimes \overline{x_{3 k}^{b(k)}}\right), \\
& 1 \leqq i \leqq j \leqq \lambda(2), \quad 1 \leqq k \leqq \lambda(3) \\
& \text { (VIII) } \quad \mu_{i j}=\sum_{h=1}^{2}\left(\begin{array}{c}
a(i) \\
h
\end{array}\right)\left(\stackrel{h}{\vee} \bar{x}_{2 i} \otimes \bar{x}_{4 j}\right)-\left(\overline{x_{2 i}^{a(i)}} \otimes \bar{x}_{4 j}\right), \\
& 1 \leqq i \leqq \lambda(2), \quad 1 \leqq j \leqq \lambda(4) \\
& \widetilde{\mu}_{i j}=c(j)\left(\bar{x}_{2 i} \otimes \bar{x}_{4 j}\right)-\left(\bar{x}_{2 i} \otimes \overline{x_{4 j}^{c(j)}}\right), \quad 1 \leqq i \leqq \lambda(2), \quad 1 \leqq j \leqq \lambda(4) \\
& \text { (IX) } \quad \eta_{i j}=b(i)\left(x_{3 i} \vee x_{3 j}\right)-\left(x_{3 j} \otimes \overline{x_{3 i}^{b(i)}}\right), \quad 1 \leqq i \leqq j \leqq \lambda(3) \\
& \tilde{\eta}_{i j}=b(j)\left(\bar{x}_{3 i} \vee \bar{x}_{3 j}\right)-\left(\bar{x}_{3 i} \otimes \overline{x_{3 j}^{b(j)}}\right), \quad 1 \leqq i<j \leqq \lambda(3) .
\end{aligned}
$$

We would like to prove that the additive abelian group $\Delta^{(4)}(G) / \Delta^{(7)}(G)$ is isomorphic to $\left\{W_{(4)}^{*} \oplus W_{(5)}^{*} \oplus W_{(6)}\right\} / R$.

For simplicity, we denote by $N$ the additive subgroup of $\Delta^{(4)}(G)$ generated by all basic products $P(\alpha)$ with $W^{*}(\alpha) \geqq 6$.

Then we have the following series of lemmas for the map $\psi$. We omit the straightforward proofs.

Lemma 4.1. For $i=4,5$ and 6 , and any $t \in T$, we have

$$
\psi\left(t\left(x_{i j}-1\right)^{a(i j)}\right) \equiv 0 \quad \bmod R, \quad 1 \leqq j \leqq \lambda(i) .
$$

Lemma 4.2. For $k=3,4$ and 5 and any $t \in T$, we have $\psi\left(t \prod_{j=1}^{k}\left(x_{2 i_{j}}-1\right)\right) \equiv \bar{x}_{2 i_{1}} \vee \bar{x}_{2 i_{2}} \vee \cdots \vee \bar{x}_{2 i_{k}} \quad \bmod R, \quad 1 \leqq i_{1} \leqq i_{2} \leqq \cdots \leqq i_{k} \leqq \lambda(2)$.

LEMMA 4.3. For any $i$ and $j$ with $1 \leqq i \leqq j \leqq \lambda(3)$, we have

$$
\psi\left(t\left(x_{3 i}-1\right)\left(x_{3 j}-1\right)\right) \equiv \bar{x}_{3 i} \vee \bar{x}_{3 j} \quad \bmod R, \quad t \in T
$$


LEMMA 4.4. For $k=3$ and 4 , any $i, j$ with $1 \leqq i \leqq j \leqq \lambda(2)$, and any $l$ with $1 \leqq l \leqq \lambda(k)$, we have

$$
\psi\left(t\left(x_{2 i}-1\right)\left(x_{2 j}-1\right)\left(x_{k l}-1\right)\right) \equiv\left(\bar{x}_{2 i} \vee \bar{x}_{2 j}\right) \otimes \bar{x}_{k l} \quad \bmod R, \quad t \in T .
$$

Lemma 4.5. For any $i$ with $1 \leqq i \leqq \lambda(2)$, and any $j, k$ with $1 \leqq j \leqq k \leqq \lambda(3)$, we have

$$
\psi\left(t\left(x_{2 i}-1\right)\left(x_{3 j}-1\right)\left(x_{3 k}-1\right)\right) \equiv \bar{x}_{2 i} \otimes\left(\bar{x}_{3 j} \vee \bar{x}_{3 k}\right) \quad \bmod R, \quad t \in T .
$$

Lemma 4.6. For any $i, j, k$ with $1 \leqq i \leqq j \leqq k \leqq \lambda(2)$, and any $l$ with $1 \leqq l \leqq \lambda(3)$, we have

$$
\begin{aligned}
& \psi\left(t\left(x_{2 i}-1\right)\left(x_{2 j}-1\right)\left(x_{2 k}-1\right)\left(x_{3 l}-1\right)\right) \\
& \quad \equiv\left(\bar{x}_{2 i} \vee \bar{x}_{2 j} \vee \bar{x}_{2 k}\right) \otimes \bar{x}_{3 l} \bmod R, \quad t \in T .
\end{aligned}
$$

By considering the map $\psi$ on each generator of $\Delta^{(7)}(G)$ in Lemma 3.3 with $u=7$ and by using Lemmas $4.1 \sim 4.6$, we can easily show the following:

Lemma 4.7. $\psi\left(\Delta^{(7)}(G)\right)=R$.

By Lemma 4.7, the homomorphism $\psi: \Delta^{(4)}(G) \longrightarrow W_{(4)}^{*} \oplus W_{(5)}^{*} \oplus W_{(6)}$ induces a homomorphism

$$
\psi^{*}: \Delta^{(4)}(G) / \Delta^{(7)}(G) \longrightarrow\left\{W_{(4)}^{*} \oplus W_{(5)}^{*} \oplus W_{(6)}\right\} / R
$$

by $\psi^{*}\left(u+\Delta^{(7)}(G)\right)=\psi(u)+R, u \in \Delta^{(4)}(G)$.

Conversely, we define a homomorphism $\phi^{*}:\left\{W_{(4)}^{*} \oplus W_{(5)}^{*} \oplus W_{(6)}\right\} / R \rightarrow$ $\Delta^{(4)}(G) / \Delta^{(7)}(G)$ by defining an element of $\Delta^{(4)}(G) / \Delta^{(7)}(G)$ on each of Z-basis of $W_{(4)}^{*}, W_{(5)}^{*}, W_{(6)}$ such that $\psi^{*} \cdot \phi^{*}$ is the identity map on $\left\{W_{(4)}^{*} \oplus W_{(5)}^{*} \oplus W_{(6)}\right\} / R$, and $\phi^{*} \cdot \psi^{*}$ is one on $\Delta^{(4)}(G) / \Delta^{(7)}(G)$ by Lemmas $4.1 \sim 4$.6. Hence we get the following:

Proposition 4.8. The additive group $\Delta^{(4)}(G) / \Delta^{(7)}(G)$ is isomorphic to $\left\{W_{(4)}^{*} \oplus W_{(5)}^{*} \oplus W_{(6)}\right\} / R=\left\{W_{(4)}^{*}(G) \oplus W_{(5)}^{*}(G) \oplus W_{(6)}(G)\right\} / R$.

To calculate the exponent of $D_{(7)}(G) / G_{7}$, we may assume $G_{7}=1$, namely, $G$ has the lower central series

$$
G=G_{1} \supset G_{2} \supset G_{3} \supset G_{4} \supset G_{5} \supset G_{6} \supset G_{7}=1 .
$$

We define a homomorphism $\Phi: G_{4} \rightarrow \Delta^{(4)}(G) / \Delta^{(7)}(G)$ by $\Phi(x)=x-1+$ $\Delta^{(7)}(G), x \in G_{4}$. Then

$$
\operatorname{Ker} \Phi=G_{4} \cap\left(1+\Delta^{(7)}(G)\right)=G \cap\left(1+\Delta^{(7)}(G)\right)=D_{(7)}(G),
$$


since $D_{(4)}(G)=G_{4}$. Here if we identify $\Delta^{(4)}(G) / \Delta^{(7)}(G)$ with $\left\{W_{(4)}^{*} \oplus W_{(5)}^{*} \oplus\right.$ $\left.W_{(6)}\right\} / R$ then we have

$$
\operatorname{Ker} \Phi=\left\{x \in G_{4} \mid \psi(x-1) \in R\right\}
$$

Since $G_{4}$ is abelian, we may write the operation of $G_{4}$ additively. Let $x=$ $\sum_{1 \leqq i \leqq \lambda(4)} r_{i} x_{4 i}+\sum_{1 \leqq j \leqq \lambda(5)} s_{j} x_{5 j}+\sum_{1 \leqq k \leqq \lambda(6)} t_{k} x_{6 k}$ be any element of Ker $\Phi$. Then it follows that

$$
x-1 \equiv \sum_{i} r_{i}\left(x_{4 i}-1\right)+\sum_{j} s_{j}\left(x_{5 j}-1\right)+\sum_{k} t_{k}\left(x_{6 k}-1\right) \quad \bmod N,
$$

and hence

$$
\psi(x-1)=\sum_{i} r_{i} \bar{x}_{4 i}+\sum_{j} s_{j} \bar{x}_{5 j}+\sum_{k} t_{k} \bar{x}_{6 k} .
$$

On the other hand, since $\psi(x-1)$ belongs to $R, \psi(x-1)$ is a $\mathbf{Z}$-linear combination of the elements of the relations (I) (IX). Denote the coefficients of the relations $(\mathrm{I}) \sim(\mathrm{IX})$ by $u_{i}(1 \leqq i \leqq \lambda(4)), u_{i}^{\prime}(1 \leqq i \leqq \lambda(5)), u_{i j}(1 \leqq i \leqq j \leqq \lambda(2)), u_{i j k}(1 \leqq$ $i \leqq j \leqq k \leqq \lambda(2)), \widetilde{u}_{i j k}(1 \leqq i<j \leqq k \leqq \lambda(2)), \widetilde{\widetilde{u}}_{i j k}(1 \leqq i \leqq j<k \leqq \lambda(2))$, $u_{i j k l}(1 \leqq i \leqq j \leqq k \leqq l \leqq \lambda(2)), \widetilde{u}_{i j k l}(1 \leqq i<j \leqq k \leqq l \leqq \lambda(2)), \widetilde{\widetilde{u}}_{i j k l}(1 \leqq i \leqq$ $j<k \leqq l \leqq \lambda(2)), \widetilde{\widetilde{u}}_{i j k l}(1 \leqq i \leqq j \leqq k<l \leqq \lambda(2)), v_{i j}(1 \leqq i \leqq \lambda(2), 1 \leqq j \leqq$ $\lambda(3)), \widetilde{v}_{i j}(1 \leqq i \leqq \lambda(2), 1 \leqq j \leqq \lambda(3)), v_{i j k}(1 \leqq i \leqq j \leqq \lambda(2), 1 \leqq k \leqq \lambda(3))$, $\widetilde{v}_{i j k}(1 \leqq i<j \leqq \lambda(2), 1 \leqq k \leqq \lambda(3)), \widetilde{\widetilde{v}}_{i j k}(1 \leqq i \leqq j \leqq \lambda(2), 1 \leqq k \leqq \lambda(3)), w_{i j}(1 \leqq$ $i \leqq \lambda(2), 1 \leqq j \leqq \lambda(4)), \widetilde{w}_{i j}(1 \leqq i \leqq \lambda(2), 1 \leqq j \leqq \lambda(4)), w_{i j}^{\prime}(1 \leqq i \leqq j \leqq \lambda(3))$, ${\widetilde{w^{\prime}}}_{i j}(1 \leqq i<j \leqq \lambda(3))$, respectively.

Then comparing the coefficients of $\bar{x}_{4 i}(1 \leqq i \leqq \lambda(4)), \bar{x}_{5 j}(1 \leqq j \leqq \lambda(5))$ and $\bar{x}_{6 k}(1 \leqq k \leqq \lambda(6))$, we have

$$
\begin{aligned}
r_{i}= & u_{i} c(i), \quad 1 \leqq i \leqq \lambda(4), \\
s_{j}= & -\sum_{1 \leqq i \leqq \lambda(4)} u_{i} c_{i j}+u_{j}^{\prime} d(j)-\sum_{1 \leqq h<i \leqq \lambda(2)} u_{h i} \frac{a(i)}{a(h)} \alpha_{j}^{(h i)}, \quad 1 \leqq j \leqq \lambda(5) \\
t_{k}= & -\sum_{1 \leqq i \leqq \lambda(4)} u_{i} c_{i k}^{\prime}-\sum_{1 \leqq i \leqq \lambda(5)} u_{i}^{\prime} d_{i k}-\sum_{1 \leqq i<j \leqq \lambda(2)} u_{i j} \frac{a(j)}{a(i)} \alpha_{k}^{(i j)^{\prime}} \\
& -\sum_{\substack{1 \leqq i \leqq \lambda(2) \\
1 \leqq j \leqq \lambda(3)}} v_{i j}\left(\sum_{j<h} a_{i h} \delta_{k}^{(h j)}\right), \quad 1 \leqq k \leqq \lambda(6)
\end{aligned}
$$

and hence

$$
\left.x=-\sum_{1 \leqq i<j \leqq \lambda(2)} u_{i j} \frac{a(j)}{a(i)}\left[x_{2 i}^{a(i)}, x_{2 j}\right]-\sum_{\substack{1 \leqq i \leqq \lambda(2) \\ 1 \leqq j \leqq \lambda(3)}} v_{i j}\left(\sum_{j<k} a_{i k}\right)\left[x_{3 k}, x_{3 j}\right]\right) .
$$


Further, comparing the coefficients of $\stackrel{2}{\vee}^{2} \vee \bar{x}_{2 j}(1 \leqq i \leqq j \leqq \lambda(2)), \bar{x}_{2 i} \vee{ }^{2} \bar{x}_{2 j}$ $(1 \leqq i<j \leqq \lambda(2)), \bar{x}_{2 i} \otimes \bar{x}_{3 k}(1 \leqq i \leqq \lambda(2), 1 \leqq k \leqq \lambda(3)), \bar{x}_{2 i} \otimes \bar{x}_{4 l} \quad(1 \leqq i \leqq$ $\lambda(2), 1 \leqq l \leqq \lambda(4)), \bar{x}_{3 j} \vee \bar{x}_{3 k}(1 \leqq j<k \leqq \lambda(3))$, we have

$$
\begin{aligned}
2 \sum_{i<j} u_{i j} \frac{a(j)}{a(i)}\left[x_{2 i}^{a(i)}, x_{2 j}\right] & =\sum_{i, k} v_{i k}\left[x_{2 i}^{a(i)}, x_{3 k}\right] . \\
\sum_{i, k} v_{i j}\left[x_{2 i}^{a(i)}, x_{3 k}\right] & =\sum_{j<k}\left(\sum_{i} v_{i k} a_{i j}-\sum_{i} v_{i j} a_{i k}\right)\left[x_{3 j}, x_{3 k}\right] \\
& =-2 \sum_{j<k}\left(\sum_{i} v_{i j} a_{i k}\right)\left[x_{3 j}, x_{3 k}\right] \\
& =-2 \sum_{i, j} v_{i j}\left(\sum_{j<k} a_{i k}\left[x_{3 j}, x_{3 k}\right]\right) .
\end{aligned}
$$

Therefore we have

$$
\begin{aligned}
2 x & =-2 \sum_{i<j} u_{i j} \frac{a(j)}{a(i)}\left[x_{2 i}^{a(i)}, x_{2 j}\right]-2 \sum_{i, j} v_{i j}\left(\sum_{j<k} a_{i k}\left[x_{3 k}, x_{3 j}\right]\right) \\
& =-\sum_{i, k} v_{i k}\left[x_{2 i}^{a(i)}, x_{3 k}\right]-2 \sum_{i, j} v_{i j}\left(\sum_{j<k} a_{i k}\left[x_{3 k}, x_{3 j}\right]\right)=0 .
\end{aligned}
$$

Thus the exponent of $D_{(7)}(G) / G_{7}$ divides 2 .

Thus we have the following:

Theorem A. If $G$ is any finite group, then the exponent of $D_{(7)}(G) / G_{7} d i$ vides 2 .

COROLlary. If $G$ is a finite $p$-group with $p \neq 2$, then $D_{(7)}(G)=G_{7}$.

Acknowledgement. We would like to thank the referee for many helpful comments which have made the paper much conciser.

\section{References}

[ 1 ] N.D. Gupta, The dimension subgroup conjecture, Bull. London Math. Soc., 22 (1990), $453-456$.

[ 2 ] T.C. Hurley and S.K. Sehgal, The Lie dimension subgroup conjecture, J. Algebra, 143 (1991), 46-56.

[ 3 ] G. Losey, N-series and filtration of the augmentation ideal, Canad. J. Math., 26 (1973), 962-977.

[4] R. Sandling, The dimension subgroup problem, J. Algebra, 21 (1972), 216-231.

[ 5 ] K-I. Tahara, On the structure of $Q_{3}(G)$ and the fourth dimension subgroup, Japan. J. Math., 3 (1977), 381-394. 
[ 6 ] K-I. Tahara, The augmentation quotients of group rings and the fifth dimension subgroups, J. Algebra, 71 (1981), 141-173.

\author{
Department of \\ Mathematical Science \\ Aichi University of EDUCATION \\ KARIYA 448, JAPAN
}

